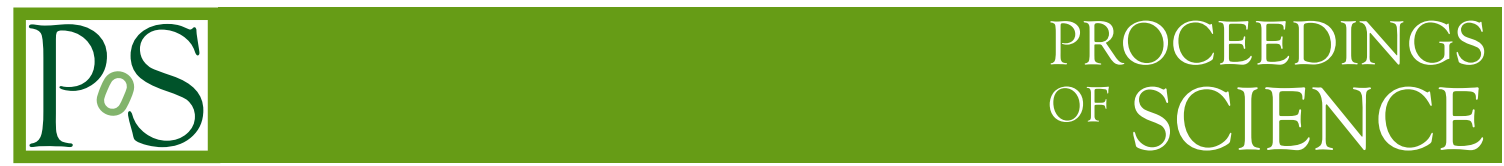

\title{
First $\mathrm{LHCb}$ results from $\mathrm{PbPb}$ collisions at $5.02 \mathrm{TeV}$
}

\section{Giulia Manca ${ }^{a * *}$ (on behalf of the LHCb collaboration)}

${ }^{a}$ Università degli studi di Cagliari and Istituto Nazionale di Fisica Nucleare,

Cittadella Universitaria di Monserrato, 09042 Monserrato (CA),Italy

E-mail: giulia.manca@cern.ch

The measurements of $\mathrm{J} / \psi$ production in peripheral and ultra-peripheral lead-lead collisions are presented using data collected by the LHCb detector in 2015 and 2018. The results are compared with several theoretical predictions.

*** The European Physical Society Conference on High Energy Physics (EPS-HEP2021), ***

*** 26-30 July 2021 ***

*** Online conference, jointly organized by Universität Hamburg and the research center DESY ***

\footnotetext{
* Speaker
}

The contact author acknowledges support from the European Research Council (ERC) through the project EXPLORINGMATTER, founded by the ERC through a ERC-Consolidator-Grant, GA 647390. 


\section{Introduction}

In the last years the LHCb experiment has provided novel inputs to heavy-ion physics by exploiting some of its unique features through the studies of $p p, p \mathrm{~Pb}, \mathrm{PbPb}$ and fixed target collisions at LHC energies for pseudorapidity $\eta$ between 2 and 5 . These measurements cover a kinematic range complementary to the ones of the other major LHC experiments, and can often reach higher precisions thanks to the excellent vertexing and particle identification capabilities of LHCb, allowing exclusive measurements of open and hidden charm states. Production measurements in this unique kinematic range provide crucial constraints on models of key interest in the field. In these proceedings, the first results of $\mathrm{J} / \psi$ production in peripheral and ultra-peripheral $\mathrm{PbPb}$ collisions are presented and compared with the available theoretical models.

\section{Detector and simulation samples}

The $\mathrm{LHCb}$ detector $[1,2]$ is a fully instrumented single-arm spectrometer in the forward region covering a pseudorapidity acceptance of $2<\eta<5$, providing a high tracking momentum resolution down to very low transverse momentum $\left(p_{\mathrm{T}}\right)$ and precise vertex reconstruction capability. Table 1 summarises the heavy-ion data samples collected by LHCb in Run 1 and Run 2. Simulation is used in the determination of efficiencies. The $p \mathrm{~Pb}$ and $\mathrm{PbPb}$ collisions are simulated with EPOS-LHC [3] and STARlight [4] and the dimuon decays with EvtGen [5] or Pythia 8.1 [6] in $p p$ collisions, where the proton beam energy is equal to the nucleon beam energy in $p \mathrm{~Pb}$ and $\mathrm{PbPb}$ collisions. The interaction of the generated particles with the detector and its response are implemented using the Geant4 toolkit [7]. In the following sections, the first results of LHCb using the $\mathrm{PbPb}$ data are presented.

\section{3. $\mathrm{J} / \psi$ photo-production in ultra-peripheral $\mathrm{PbPb}$ collisions}

A collision is called ultra-peripheral when the two colliding nuclei barely miss each other, such that the impact parameter is larger than the sum of their two radii. Despite the fact that they do not physically touch each other, they can however interact by exchanging a photon, and this interaction can be greatly enhanced compared to $p p$ by the strong electromagnetic field of the nucleus. The interaction can be coherent ( $\gamma$ interacts with the nucleus as a whole) or incoherent ( $\gamma$ interacts with one or more of the nucleons in the nucleus). The measurement of the rate of coherent charmonia production can be extremely important to constrain the gluon PDFs, while the ratio of production of different charmonia states can discriminate on inputs in certain color dipole models [8].

\begin{tabular}{c|cccc|cc|} 
year & \multicolumn{2}{|c}{2013} & \multicolumn{2}{c|}{2016} & 2015 & 2018 \\
\hline$\sqrt{s_{\mathrm{NN}}}$ & \multicolumn{2}{|c|}{$5.02 \mathrm{TeV}$} & \multicolumn{2}{c|}{$8.16 \mathrm{TeV}$} & $5.02 \mathrm{TeV}$ \\
\hline & $p \mathrm{~Pb}$ & $\mathrm{~Pb} p$ & $p \mathrm{~Pb}$ & $\mathrm{~Pb} p$ & $\mathrm{PbPb}$ \\
$\mathcal{L}$ & $1.1 \mathrm{nb}^{-1}$ & $0.5 \mathrm{nb}^{-1}$ & $13.6 \mathrm{nb}^{-1}$ & $20.8 \mathrm{nb}^{-1}$ & $10 \mu b^{-1}$ & $228 \mu b^{-1}$
\end{tabular}

Table 1: Heavy-ion data samples collected by the LHCb experiments in Run 1 and Run 2. 
The $\mathrm{J} / \psi$ coherent cross section has been measured as a function of rapidity in $\mathrm{PbPb}$ collisions at $\mathrm{LHCb}$ using the 2015 dataset at $\sqrt{s_{\mathrm{NN}}}=5.02 \mathrm{TeV}$, according to the formula

$$
\frac{d \sigma_{\text {coherent }}^{\mathrm{J} / \psi}}{d y}=\frac{N_{\text {coherent }}^{\mathrm{J} / \psi}}{\epsilon_{\text {tot }} \cdot \mathcal{L} \cdot \Delta y \cdot B\left(\mathrm{~J} / \psi \rightarrow \mu^{+} \mu^{-}\right)},
$$

where $N_{\text {coherent }}^{\mathrm{J} / \psi}$ is the number of $\mathrm{J} / \psi$ signal events, $\epsilon_{\text {tot }}$ is the total efficiency, $\mathcal{L}$ is the integrated luminosity, $\Delta y$ is the rapidity interval and $B\left(\mathrm{~J} / \psi \rightarrow \mu^{+} \mu^{-}\right)$is the $\mathrm{J} / \psi \rightarrow \mu^{+} \mu^{-}$branching ratio. The events are selected by requiring two long tracks (the muons) and nothing else in the detector, where the new HERSCHEL detector [9] is used for this requirement. The signal candidate events are then determined by a fit on the dimuon invariant mass in the kinematic range $2.0<\eta^{\mu}<4.5$, $p_{\mathrm{T}}^{\mu}>700 \mathrm{MeV} / c, p_{\mathrm{T}}^{\mu \mu}>1 \mathrm{GeV} / c$ and $\left|\Delta \phi_{\mu \mu}\right|>0.9 \pi$, shown in Fig. 1, left. The coherent component is separated by the incoherent one through a fit on the momentum of the dimuon system shown in Fig. 1, right. The coherent $J / \psi$ cross section as a function of rapidity is shown in Fig. 2, where the
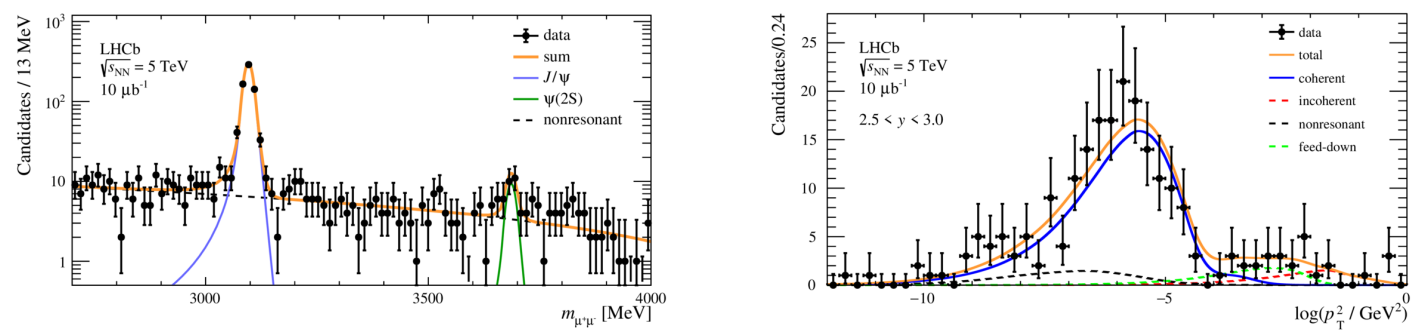

Figure 1: (Left) Dimuon invariant mass after all selection where the data (dots) are overlayed to the results of the fit (histogram) and (right) transverse momentum distribution of the same events. The coherent contribution is well visible in the low- $p_{\mathrm{T}}$ region in the region of $\log \left(p_{\mathrm{T}}{ }^{2} / \mathrm{GeV} / c^{2}\right)$ between -10 and -4 .

results are compared to several theoretical models using perturbative QCD calculations [10] and color dipole models $[8,11,12]$. The measurement has the potential to allow great discrimination
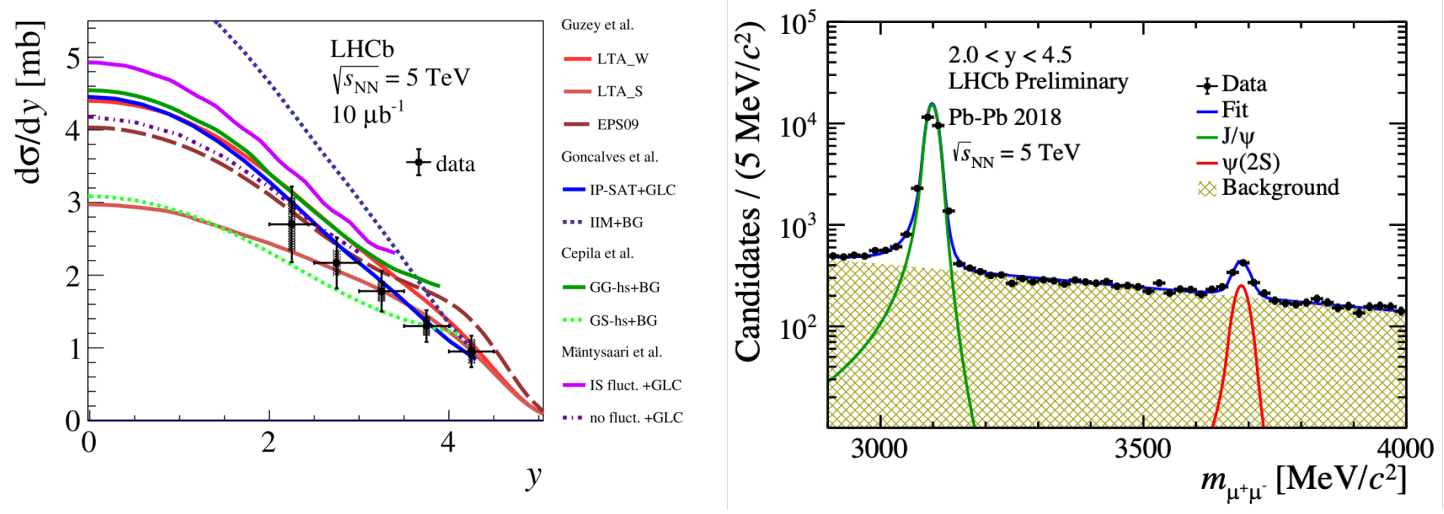

Figure 2: (Left) Differential $\mathrm{J} / \psi$ cross section measurement as a function of rapidity in UPC PbPb collisions. The data (dots) are compared to several theoretical prediction discussed in the text. (Right) Dimuon invariant mass in $2018 \mathrm{PbPb}$ collisions.

among the different models in the region at lower rapidity where the differences between the theories 
are more pronounced. More precise results are expected by the analysis of the $2018 \mathrm{PbPb}$ sample, roughly 20 times larger; the dimuon invariant mass for this sample is shown in Fig. 2, where both the $\mathrm{J} / \psi$ and $\psi(2 S)$ are clearly visible [13].

\section{Study of $\mathrm{J} / \psi$ production in peripheral $\mathrm{PbPb}$ collisions}

When the two nuclei barely touch each other, the collision is called "peripheral", and it is defined by an impact parameter $b$ smaller than the sum of the radii of the nuclei (see Fig. 3). This type of process is very interesting as it is possible to identify both the photo-production and the hadronic production of the $\mathrm{J} / \psi$ mesons. In $\mathrm{LHCb}$ the production of prompt $\mathrm{J} / \psi$ decaying to two muons is studied as a function of rapidity, transverse momentum and centrality of the event.

\subsection{Centrality determination}

The centrality $C$ of the event is a quantity related to the impact parameter of the collisions, the smaller $b$ the higher $C$. It is defined as percentile of the inelastic $\mathrm{PbPb}$ cross-section as a function of the impact parameter $b$, and in LHCb $C$ is evaluated [14] using a Glauber model to derive quantities as the number of particle participating to the event $\left(N_{\text {part }}\right)$ and $b$, from the energy deposited in the electromagnetic calorimeter (ECal), which is used as proxy ( see Fig. 3). In the LHCb-RunII only
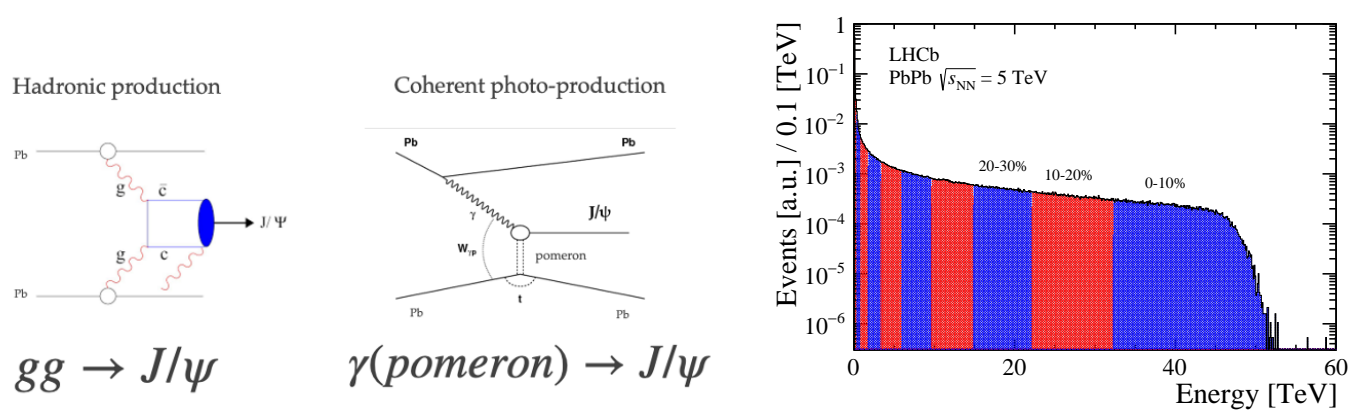

Figure 3: (Left) Production of $\mathrm{J} / \Psi$ in peripheral collisions. (Right) ECal distribution in bins of centrality in $2018 \mathrm{PbPb}$ collisions.

centralities down to $60-70 \%$ can be reached, due to the saturation of the tracking system.

\subsection{Analysis}

The main challenge of the analysis [15] is to separate the $\mathrm{J} / \psi$ 's from coherent photoproduction, mainly at low $p_{\mathrm{T}}$, from the hadronically produced one, populating the higher- $p_{\mathrm{T}}$ region. In order to do this, after a preselection based on the track quality and the distance from the primary vertex (to reject the non-prompt component), the signal events are selected through the combined requirement on the invariant mass of the two muons to be in the mass window of the $\mathrm{J} / \psi$ meson and on the $p_{\mathrm{T}}$ of the two muons. In the $p_{\mathrm{T}}$ distribution shown in Fig. 4 the two components can be clearly identified. The selected events, corrected for the geometric acceptance and reconstruction and selection efficiency, are used to measure the yield of $\mathrm{J} / \psi$ photoproduction, defined as

$$
\frac{d Y_{i}^{\mathrm{J} / \psi}}{d y}=\frac{N_{i}^{\mathrm{J} / \psi}}{N_{i}^{M B} \epsilon_{i}^{t o t} \cdot \Delta y \cdot B\left(\mathrm{~J} / \psi \rightarrow \mu^{+} \mu^{-}\right)} .
$$



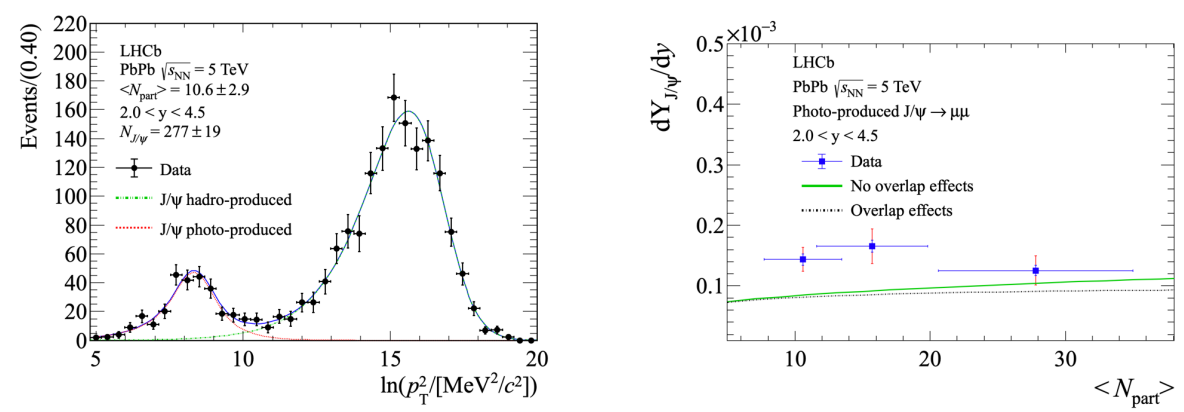

Figure 4: (Left) Distribution of $\mathrm{J} / \psi p_{\mathrm{T}}$ in peripheral collisions, where the two production components are clearly visible. (Right) Yield of photoproduced $\mathrm{J} / \psi$ integrated over $p_{\mathrm{T}}$ and $y$ as a function of $N_{\text {part }}$.

where $N_{i}^{\mathrm{J} / \psi}, N_{i}^{M B}$ and $\epsilon_{i}^{\text {tot }}$ are the number of signal and minimum bias events and the total efficiency in the $i$-th centrality interval respectively, $\Delta y$ is the rapidity interval and $B\left(\mathrm{~J} / \psi \rightarrow \mu^{+} \mu^{-}\right)$ is the $\mathrm{J} / \psi \rightarrow \mu^{+} \mu^{-}$branching ratio. The yield is measured as a function of $y, p_{\mathrm{T}}, N_{\text {part }}$ as well as integrated over $y$ and $p_{\mathrm{T}}$, as shown in Fig.4. The results are compared to two theoretical models $[16,17]$ and found to be consistent.

\section{Conclusions and outlook}

$\mathrm{LHCb}$ is in a unique position to perform heavy-ion physics in the region of forward rapidity with a precision unreachable by other current experiments, crucial to constrain the theoretical models. The first results in $\mathrm{PbPb}$ collisions estabilish $\mathrm{LHCb}$ as a key player in the game, laying the foundation for more inclusive results expected in the upcoming Run 3, when higher values of centralities will be accessible.

\section{References}

[1] LHCв COLLABORATION collaboration, The LHCb detector at the LHC, JINST 3 (2008) S08005.

[2] LHCв collaboration collaboration, LHCb detector performance, Int. J. Mod. Phys. A30 (2015) 1530022 LHCB-DP-2014-002, CERN-PH-EP-2014-290, [1412 . 6352].

[3] T. Pierog, I. Karpenko, J.M. Katzy, E. Yatsenko and K. Werner, EPOS LHC: Test of collective hadronization with data measured at the CERN Large Hadron Collider, Phys. Rev. C92 (2015) 034906 [1306.0121].

[4] S. Klein, J. Nystrand, J. Seger, Y. Gorbunov and J. Butterworth, Starlight: A monte carlo simulation program for ultra-peripheral collisions of relativistic ions, Computer Physics Communications 212 (2017) 258.

[5] D.J. Lange, The EvtGen particle decay simulation package, Nucl. Instrum. Meth. A462 (2001) 152. 
[6] T. Sjöstrand, S. Mrenna and P. Skands, A brief introduction to PYTHIA 8.1, Comput. Phys. Commun. 178 (2008) 852 [0710.3820].

[7] Geant4 collaboration collaboration, Geant4 developments and applications, IEEE Trans.Nucl.Sci. 53 (2006) 270.

[8] H. Mäntysaari and B. Schenke, Probing subnucleon scale fluctuations in ultraperipheral heavy ion collisions, Phys. Lett. B772 (2017) 832 [1703. 09256].

[9] K. Carvalho Akiba et al., The HeRSCheL detector: high-rapidity shower counters for LHCb, JINST 13 (2018) P04017 LHCb-DP-2016-003, [1801 . 04281].

[10] V. Guzey, E. Kryshen and M. Zhalov, Coherent photoproduction of vector mesons in ultraperipheral heavy ion collisions: Update for run 2 at the CERN Large Hadron Collider, Phys. Rev. C93 (2016) 055206 [1602.01456].

[11] V.P. Gonçalves, M.V.T. Machado, B. Moreira, F.S. Navarra and G.S. dos Santos, Color dipole predictions for the exclusive vector meson photoproduction in $\mathrm{pp}, \mathrm{pPb}$, and $\mathrm{PbPb}$ collisions at run 2 LHC energies, Phys. Rev. D96 (2017) 094027 [1710 . 10070].

[12] J. Cepila, J.G. Contreras and M. Krelina, Coherent and incoherent $\mathrm{J} / \Psi$ photonuclear production in an energy-dependent hot-spot model, Phys. Rev. C97 (2018) 024901 [1711.01855].

[13] LHCв collaboration, “https://twiki.cern.ch/twiki/bin/view/LHCb/LHCbPlotsQM2019."

[14] R. Aaij et al., Centrality determination in heavy-ion collisions with the LHCb detector, LHCb-DP-2021-001.

[15] LHCв COllaboration collaboration, Study of J/ $\psi$ photo-production in lead-lead peripheral collisions at $\sqrt{s_{N N}}=5 \mathrm{TeV}, 2108.02681$ LHCb-PAPER-2020-043, CERN-EP-2021-122, [2108.02681].

[16] W. Zha, S.R. Klein, R. Ma, L. Ruan, T. Todoroki, Z. Tang et al., Coherent j/ $\psi$ photoproduction in hadronic heavy-ion collisions, Phys. Rev. C 97 (2018) 044910.

[17] W. Zha, L. Ruan, Z. Tang, Z. Xu and S. Yang, Double-slit experiment at fermi scale: Coherent photoproduction in heavy-ion collisions, Phys. Rev. C 99 (2019) 061901. 